\title{
Reading Performance and Academic Achievement in Early Childhood Bilingual and Monolinguals
}

\author{
Abootaleb Seadatee-Shamir ${ }^{*}$, Zohreh Siavoshi² \\ ${ }^{1}$ Counseling and Educational Psychology Department, Farhangian University, Bojnord, Iran \\ ${ }^{2}$ Azad University of Bojnord, Science and Research Branch of North Khorasan, Bojnord, Iran \\ Email: seadatee@yahoo.com
}

Received 1 April 2014; revised 4 May 2014; accepted 12 May 2014

Copyright (C) 2014 by authors and Scientific Research Publishing Inc.

This work is licensed under the Creative Commons Attribution International License (CC BY). http://creativecommons.org/licenses/by/4.0/

c) (i) Open Access

\section{Abstract}

Relationship between reading performance and academic achievement is theoretically predictable. However, this relationship could be higher among early childhood bilingual (ECBL) students than monolingual peers. For evaluating this claim, two groups of randomly selected female second graders (150 Farsi speaking and 150 Farsi and Turkish speaking) were tested and compared on these characteristics. The measures used were Kormi-Nouri and associates' tests of reading performance. Moreover, academic achievement was defined to be the average of scores obtained by students at their final school exams in Math, Science and Farsi. Analysis of regression was then performed to determine which construct served as the best predictor of academic achievement in both groups. Based on the results, ECBL girls were superior to their monolingual (ML) counterparts in all three areas. Findings also show that reading performance was a strong predictor of school achievement for both groups.

\section{Keywords}

Reading Performance, Academic Achievement, Early Childhood Bilinguals

\section{Introduction}

Several studies show that the phonological component of memory plays a major role in second language acquisition (e.g., Thorn \& Gathercole [1]). Performance differences between monolingual (ML) and (ECBL) on measures of academic achievement are often attributed to limitations in reading (e.g., De Jong [2], Siegel \&

\footnotetext{
${ }^{*}$ Corresponding author.
}

How to cite this paper: Seadatee-Shamir, A. and Siavoshi, Z. (2014) Reading Performance and Academic Achievement in Early Childhood Bilingual and Monolinguals. Open Journal of Applied Sciences, 4, 347-353. 
Ryan [3], Swanson [4]). However, there is no consensus about the nature of reading or, more specifically, about this issue that which components of academic achievement are most affected by deficiencies in reading ability.

One model tested, consistent with the work of Crain et al. [5] and others (Stanovich \& Siegel [6]) suggests that poor readers are deficient in setting up phonological structures. For example, reading comprehension is compromised because inefficient phonological analysis creates a "bottleneck" that constricts information flow to higher levels of processing. Executive processing in their model has the primary task of relaying the results of lower-level linguistic analyses upward through the language system. Readers' word recognition difficulties relate to problems in the articulatory system. In addition, comprehension difficulties follow automatically from limitations in word skills and, therefore, correlations between academic achievement and reading comprehension should be significantly weakened if measures reflective of the articulatory system are partialed from the analysis. This is, if ML readers' problems in reading (whether at the word recognition or comprehension level) and WM are moderated by the articulatory system, then the relationship between reading and academic achievement should diminish when measures of the articulatory system (e.g., articulatory speed) are partialed from the analysis.

Despite a growing literature on bilingual children, there are few studies that specifically address ECBL in a multi-language community. Consistent with this idea, we wish to investigate the predictive role of WMC and reading performance in academic achievement of ECBL and ML students.

Consistent with these results, there is extensive evidence showing bilingualism advantages in executive functioning using tasks based on response conflict, switching, and flexibility (e.g., Bialystok \& Shapero [7]; Carlson \& Meltzoff [8]; Martin-Rhee \& Bialystok [9]). Furthermore, other widely accepted studies (Bialystok [10] [11] of bilingualism development suggest that although the basic features of the language acquisition process in both groups are very similar but bilinguals outperform their ML peers in different cognitive tasks which leads to academic achievement. It is mostly because, while ML children confront only one form of linguistic input, bilinguals confront two forms and two channels of linguistic input. Consequently, they have higher levels of met linguistic awareness when compared to their ML peers. So they outperform their ML peers in academic tasks which need broad and flexible knowledge of linguistic and semantic. In addition, they attain relatively high levels of phonological awareness precisely because of the experience with two language systems and the frequent attention to the phototoxic aspects of language. That's why they are more attentive and experienced in academic tasks like mathematic which need high concentration and flexibility.

In spite of extensive body of research which accepted the superiority of bilinguals compared with monolinguals but there are some shortcomings concerning ECBl which mainly include deficits in lexical access and code switching. ECBl children often interject words from the other language when they do not know or cannot remember the equivalent, and when one language has no suitable equivalent in the other. Taeschner found that bilingual children prefer to insert elements of the other language rather than use simplified forms (Schwartz, Geva, Share \& Leikin, M. [12]).

Furthermore, Pearson's finding [13] reveals that Code-switching occurs when a child combines more than one language in a single utterance. This phenomenon is also seen in bilingual adults. Bilingual children most often engage in intrasentential code-switching, switching languages in the middle of a sentence. In addition, if a child's parents engage in code-switching in their own speech, this will affect the child's perception of the appropriateness of mixing languages.

Although, some challenges exist in this area and extensive body of previous research accepted the superiority of bilinguals in reading performance and academic achievement (e.g. [11], but to my knowledge very few studies explore the difference between ECBL and ML in Multilanguage countries like Bojnoury. The novelty of this study is that the first language of the samples of this study was formal and the second language was informal language (kurmanji). While in most studies, two languages of bilinguals are formal language. In addition, another relatively common assumption in similar studies is that academic achievement is important predictors of children's reading performance of language skills (e.g. [14] and school achievement [15]).

Finally, two questions are addressed: First, the role of reading performances in predicting academic achievement of ML and ECBL students. Second, is there a meaningful difference between two groups in two constructs? It has to be mentioned that the present study was conducted in Iran, which is a multilingual country where different groups of people such as Persian (Farsi), Kormanj, Turks, Kurds, Arabs and etc., living together. Although they all have one official language (Persian) in schools, universities, and governmental departments, etc., they use their mother tongue or second language at home and on the street. Both groups are living in Bojnord 
city (a multilingual speaking area). They used Persian as school language and ECBL student's used Turkish language as a second language.

\section{Materials/Tasks}

\subsection{Reading Performance Tasks}

Because there is no standardized reading performance test, using factor analysis, 5 tasks (Which were loaded of one factor and all clustered to form reading performance component) were selected from a battery of standardized reading comprehension tests for Iranian children (Karami Noori et al., [14]). This task is specifically designed and its psychometric properties ascertained. This test is composed of five separate and timed paper and-pencil sub tests. Participants were allowed at the range of 2 to 3 minute to complete each subtest (exact time varies for each subtest). Before administration the participant was presented with an instruction sheet followed by an answer sheet. Instruction sheet is consisting of a series of sample for every subtest. They were instructed to keep trying and do as fast as possible in each subtest within time limitation and mark the correct answer in answer sheet. Experimenter is not allowed to help participant during the administration of the task. When time expired for a subtest, participants were instructed to stop working on that subtest and begin the next. At no point, the participants were allowed to go back to work on previous sub tests. Experimenter score correct answers of each subtest independently. The reading performance score was the sum of all correct answers, across all five sub tests. Reading performance sub tests are as follows.

\subsubsection{Word Reading}

This task is consisting of three lists. Every list includes 40 words. Words are arranged according to their frequency in three levels (high, medium and low). Two-minute is considered as time limitation for each list. Participants were asked to read as much as words they can and then mark the correct answer in answer sheet. Then, experimenter scored correct answers for each list independently. The maximum score for every list is 40 and for whole subtest are 120 .

\subsubsection{Pseudo Word Reading}

This task was administered to assess phonological skills (awareness). In this subtest, participants were supposed to read 40 pseudo words according to a list presented to them from right to left and up to down. Participants were instructed not to concentrate on meaning of the words but just in reading with correct pronunciation (one point for every correct answer). Maximum score in this subtest is 30 .

\subsubsection{Text Comprehension}

This sub-test consists of three narrative story texts. Two stories are specifically designed for third grade but one story is common for all five grades of elementary students. Story texts are designed according to their interest, needs, cognitive level and their academic curriculum. Number of words in each text is between 320 to 340 . There are 8 questions for each text and four alternatives for every single question. Maximum score in this subtest is 24 . Internal consistency, as measured by Cronbach's alpha, for the 3 passages was 0.90 , indicating that these passages as a whole and the answers to the comprehension questions were consistent and reliable. The inter-reliabilities for the answers to the 24 questions of these four passages were respectively, $0.81,0.82$, and 0.80 .

\subsubsection{Phoneme Deletion}

This sub-test consists of 30 words with 2 or 3 phonemes. In the first step, experimenter read every word loudly and asks the participant to omit the expected syllable according to word list and reproduce the remaining word after him. In some words, first syllable and in some others second or last syllable was omitted. The dependent measure was the number of correct answers. Maximum score in this subtest is 30 .

\subsubsection{Letter Signs}

This sub-test consists of three letters from 32 alphabets of Persian letters. Each word which is printed with a bold font on an independent card is presented to participant. Then experimenter read each letter with a high sound and participant is asked to make as much as words, starting with this letter. Produced word is written on answer sheet by experimenter. An important point in this subtest is that every letter has two written form in Per- 
sian. So, both forms are presented in a single card and participant is instructed to make word with both forms within time limitation ( 1 minute for every letter and 3 minutes for all). One point is for every correct produced answer. Maximum score in this subtest is 30 .

\subsection{Academic Achievement}

The average of scores obtained by students at their final school exams in Math, Science, and Farsi were selected as the student's measure of academic achievement.

\section{Procedure}

A total of 400 second grade children aged between 9 and 10 years from female state elementary school of Bojnord who were rated as having average family socioeconomic status took part in the study. Three classes (nearly 25 - 30 members) were randomly selected from each school. Then they were grouped according to their self-reported ML or bilingual status. Before proceeding with task administration, informed consent was gained from the participants. They complete a researcher made form which was to distinguish between different categories of bilingualism. Then, participants were informed that the project would be conducted in two stages in one session. Each participant was tested individually. The language of experiment was Persian for all ML and BL participants. Due to the high population of the study, 5 fully instructed bilingual (Persian and Kormanji speaking) senior research collaborators (three full time and two part-time) from a local university were employed to administer the tasks. They were given five day's intensive training on the rational of the project, the reason and the design of the groups and individual tasks and specific of the administration before their field work in the schools. This assistance all had experience in working on other projects. During the interactive training sessions, also offered considerable advice on the tasks, which were further modified and fine tuned before their administration. This experienced assistance was carefully supervised by the first author of the present study to ensure high fidelity of the field work. At the first step, students last year score of final exams in Math, Science, and Farsi were gathered as their academic achievement measure.

At the next stage, information processing task were conducted. Then sub-tests of reading performance task were presented respectively. The tasks were administered in a well illuminated room, furnished with a large desk, a computer, and a chair situated in front of the desk. The experimenter sat in a chair adjacent to the computer desk with the mouse and keyboard situated in front of him to facilitate operation of the tasks. So, experimenters were present for all tasks, and participants completed the tasks individually. Following completion of all tasks, participants were debriefed regarding the purpose of the project. Participants who did not meet the criterion for tasks (multilingual and unrelated bilinguals) were not included in final data analysis.

\section{Results}

Preliminary scanning showed that all variables were practically, normally distributed; correlations matrix revealed that the five reading performance tasks and academic achievement subcomponent have consistent yet moderate correlations in the range of 0.20 - 0.62 and 0.20 - 0.66 in ML and of $0.19-0.75$ in ECBL respectively. Second, among all the reading performance tasks, phoneme deletion and letter sign have the highest $(0.92)$ and lowest (0.44) correlation in ML and letter sign and phoneme deletion have the highest (0.92) and lowest (0.17) correlation in ECBL respectively. Concerning academic achievement measures, Farsi (0.82), Math (0.21), Math (0.50) and Science (0.17) have the highest and lowest correlation coefficient in ML and ECBL respectively. Also Hierarchical regression for prediction of academic achievement has been presented in Table 1.

Hierarchical regression for prediction of academic achievement in Table 1 shows that in the first step reading

Table 1. Hierarchical regression for prediction of academic achievement.

\begin{tabular}{ccccccccc}
\hline Variable & $\mathbf{R}$ & $\mathbf{R}^{2}$ & $\mathbf{F}$ & df1 & df2 & Sig. & T & 300. \\
\hline Rp & 510. & 260. & 77.72 & 1 & 300 & 001.0 & 260.0 \\
Lang & 520. & 310. & 50.65 & 1 & 299 & 2.2 \\
\hline
\end{tabular}

Note: Rp stands for reading performance; Lang for language; R stands for coefficient of correlation; $\mathrm{R}^{2}$ stands for coefficient of determination; $\mathbf{F}$ stands for $\mathrm{f}$ test; Df stands for degree of freedom; sig $\mathbf{F}$ stands for significance; $\beta$ stands for beta and $\mathbf{T}$ stands for $\mathrm{t}$ test. 
performance was entered to regression equation. Results show that $\mathrm{f}(72.77)$ in $0 / 001$ level with degree of freedom $(1,400)$ is significant and acquired $R^{2}$ according to these two variables is $(0.26)$. In other word, reading performance can predict (0.26) of the variance of academic achievement. In the second step language was entered to regression equation. Regression effect of two variables is $\mathrm{f}(50.65)$ in 0.001 levels with degree of freedom $(1,399)$ is significant and acquired $\mathrm{R}^{2}$ according to these two variables is $(0.31)$. It means that reading performance can predict only 0.05 of academic achievement variance. This finding shows that reading performance and language can significantly predict academic achievement respectively. ANOVA showed that ECBL group was superior to their ML counterparts in all variables except in two of the reading performance test's sub-variable (phoneme deletion and pseudo word reading).

\section{Discussion}

The purpose of this study was to determine whether there are meaningful differences between reading performance and academic achievement of ML and ECBL students. The results suggest a moderate link between these variables in both groups. As such, these data provide support for previous claims that reading performance is an essential aspect of academic achievement in bilingual student (Fontoura \& Siegel [14]; Gathercole et al., [15]; Gropper \& Tannock [16]; Moehara \& Saitos [17]). In addition, these findings have implications for classic cognitive ability studies linking reading performance to academic achievement (Andersson \& Lyxell [18]; Alloway \& Alloway [19]; Alloway [20]; Gathercole et al. [21], Swanson \& O’Connor [22]).

Findings of the present study provide support for the speculation that bilingual children had significantly higher scores on reading performance and academic achievement than ML peers (Girbau \& Schwartz [23]; Siegel [24]; Ferrari \& Palladino [25]; Carlson \& Meltzoff [26]). The results yield three clear findings on this issue. First, $\mathrm{Ml}$ readers' performance is inferior to ECBL on AC academic achievement measures, and that partialing articulation speed from these measures does not eliminate performance differences. These deficits in performance are pervasive across verbal tasks. Second, Ml readers suffer lack of experience and storage deficits independent of their readers' performance is native language. In addition, resultS show that reading experience in two languages contributes unique variance to word recognition and reading comprehension beyond that contributed by one language. Finally, in many components of academic achievement, Ml readers' are clearly inferior to ECBL readers.

Moreover, the finding concerning ECBL student reveals that, they have the capacity to develop creative meaning, association and thinking more naturally than MLs do. Consequently, they are outperforming in Math, Science and Farsi comparing to their ML counterparts. But, how well an ECBL develops its second language can vary from a child who only knows a few phrases and some very basic vocabularies, to a child who listens and understands, but cannot or perhaps will not speak, to a balanced bilingual child (who communicates in both languages) with the same command as their ML peers in both languages.

Still further findings of the present study are in line with studies suggesting that bilingual children not only better appreciate what is human versus what is cultural, but they are also more inclined to have a deeper appreciation of language. They understand at an early age that there is more than one way to label or discuss something. They understand that different labels for the same object or idea in different languages can have different connotations. They are more likely to see the creative possibilities of language and explore their own linguistic creativity (Saiegh-Haddad \& Geva [26]; Bialystok \& Feng [27]; Kohnert \& Bates [28]). Consequently they have a better performance in text comprehension than other observed variables of reading performance. The present findings also converge on the view that language is a crucial tool in forming cognition, which enables a child to organize abstract thoughts. Perfetti \& Liu [29] suggest that the internalization of two languages rather than one results in a more complex, better equipped cognition, which enables the child to alternate between the two linguistic codes (Gathercole et al. [15]). Furthermore, Kohnert et al. [30] assert that, in addition to stretching their memory and minds intellectually, learning two languages allows bilingual person to develop their instructional and cultural understanding of people beyond them. Therefore, this capability leads ECBL students to have better syntactic, semantic, and linguistic and finally school achievement than their ML peers.

Our results provide further support for the proposal in the bilingualism literature that is able to step into another culture through its language. Although some ECBL children do not have a lot of exposure to the culture of their second language, the language itself conveys much of the culture to them. Further, even if, ECBL children are not learning the language academically, but they can attain grammatical and semantic language skills 
orally through communication in their house or community. In this case, they are potentially capable of experiencing more cognitive exercise to develop their cognitive abilities. Moreover, learning two languages simultaneously helps them to extend the storage of the different vowels, sounds, letters and benefit a rich cognitive and linguistic storage in their memory.

In this case, they can improve their knowledge in different educational areas especially at school and as a result, attain a better academic achievement than their ML peers. Another important issue concerning the nature of bilingualism consistent with the finding of this study is that ECBL children have some experience seeing how different cultures cause different people to interpret completely differently the exact same circumstance. In sum, the crucial finding we wish to emphasize is that ECBL children with relatively high WMC and reading performance are more successful in academic achievement than their ML peers. Although, there are some little probable disadvantages of ECBL, which plays an important role in attaining academic achievement and other cognitive variables. But this study highlights the importance of early language skills' acquisition in two (or more) language speaking families.

\section{References}

[1] Thorn, A.S.C. and Gathercole, S.E. (1999) Language-Specific Knowledge and Short-Term Memory in Bilingual and Non-Bilingual Children. Quarterly Journal of Experimental Psychology, 52A, 303-324. http://dx.doi.org/10.1080/713755823

[2] DeJong, P.F. (1998) Working Memory Deficits of Reading Disabled Children. Journal of Experimental Child Psychology, 70, 75-96. http://dx.doi.org/10.1006/jecp.1998.2451

[3] Siegel, L.S. and Ryan, E.B. (1988) Development of Grammatical-Sensitivity, Phonological and Short-Term Memory Skills in Normally Achieving and Subtypes of Learning Disabled Children. Developmental Psychology, 24, 28-37. http://dx.doi.org/10.1037/0012-1649.24.1.28

[4] Swanson, H.L. (1989) Verbal Coding Deficits in Learning-Disabled Readers: A Multiple Stage Model. Educational Psychology Review, 1, 235-277. http://dx.doi.org/10.1007/BF01320136

[5] Crain, S., McKee, C. and Emiliani, M. (1990) Visiting Relatives in Italy. In: Frazier, L. and Villiers, J.D., Eds., Language Processing and Language Acquisition, Kluwer, Dordrecht, 335-356. http://dx.doi.org/10.1007/978-94-011-3808-6_14

[6] Stanovich, K.E. and Siegel, L.S. (1994) Phenotypic Performance Profile of Children with Reading Disabilities: A Regression-Based Test of the Phonological-Core Variable-Difference Model. Journal of Educational Psychology, 86, 24-53. http://dx.doi.org/10.1037/0022-0663.86.1.24

[7] Bialystok, E. and Shapero, D. (2005) Ambiguous Benefits: The Effect of Bilingualism on Reversing Ambiguous Figures. Developmental Science, 8, 595-604. http://dx.doi.org/10.1111/j.1467-7687.2005.00451.x

[8] Carlson, S.M. and Meltzoff, A.N. (2008) Bilingual Experience and Executive Functioning in Young Children. Developmental Science, 11, 282-298. http://dx.doi.org/10.1111/j.1467-7687.2008.00675.x

[9] Martin-Rhee, M.M. and Bialystok, E. (2008) The Development of Two Types of Inhibitory Control in Monolingual and Bilingual Children. Bilingualism: Language and Cognition, 11, 81-93. http://dx.doi.org/10.1017/S1366728907003227

[10] Bialystok, E. (1988) Levels of Bilingualism and Levels of Linguistic Awareness. Developmental Psychology, 24, 560567. http://dx.doi.org/10.1037/0012-1649.24.4.560

[11] Bialystok, E. (1997) Effects of Bilingualism and Illiteracy on Children’s Emerging Concepts of Print. Developmental Psychology, 33, 429-440. http://dx.doi.org/10.1037/0012-1649.33.3.429

[12] Schwartz, M., Geva, E., Share, D. and Leikin, M. (2007) Learning to Read in English as a Third Language: The Cross-Linguistic Transfer of Phonological Processing Skills. Written Language and Literacy, 10, 25-52. http://dx.doi.org/10.1075/wll.10.1.03sch

[13] Pearson, B. (2007) Social Factors in Childhood Bilingualism in the United States. Applied Psycholinguistics, 28, 399-410. http://dx.doi.org/10.1017/S014271640707021X

[14] Fontroura, H. and Siegel, L.S. (1995) Reading, Syntactic and Working Memory Skills of Bilingual Portuguese-English Canadian Children. Reading and Writing, 7, 139-153. http://dx.doi.org/10.1007/BF01026951

[15] Gathercole, S.E., Alloway, T.P., Willis, C. and Adams, A.-M. (2006) Working Memory in Children with Reading Disabilities. Journal of Experimental Child Psychology, 93, 265-281. http://dx.doi.org/10.1016/j.jecp.2005.08.003

[16] Gropper, R.J. and Tannock, R. (2009) A Pilot Study of Working Memory and Academic Achievement in College Students with ADHD. Journal of Attention Disorders, 12, 574-581. http://dx.doi.org/10.1177/1087054708320390 
[17] Moehara, Y. and Saitos, S. (2007) The Relationship between Processing and Storage in Working Memory Span: Not Two Sides of the Same Coin. Journal of Memory and Language, 56, 212-228. http://dx.doi.org/10.1016/j.jml.2006.07.009

[18] Andersson, U. and Lyxell, B. (2007) Working Memory Deficit in Children with Mathematical Difficulties: A General or Specific Deficit? Journal of Experimental Child Psychology, 96, 197-228. http://dx.doi.org/10.1016/j.jecp.2006.10.001

[19] Alloway, T.P. and Alloway, R.G. (2010) Investigating the Predictive Roles of Working Memory and IQ in Academic Attainment. Journal of Experimental Child Psychology, 106, 20-29. http://dx.doi.org/10.1016/j.jecp.2009.11.003

[20] Alloway, T.P. (2009) Working Memory, but Not IQ, Predicts Subsequent Learning in Children with Learning Difficulties. European Journal of Psychological Assessment, 25, 92-98. http://dx.doi.org/10.1027/1015-5759.25.2.92

[21] Gathercole, S.E., Hitch, G.J., Service, E. and Martin, A.J. (1997) Short-Term Memory and New Word Learning in Children. Developmental Psychology, 33, 966-979. http://dx.doi.org/10.1037/0012-1649.33.6.966

[22] Swanson, H.L. and O’Connor, R. (2009) The Role of Working Memory and Fluency Practice on the Reading Comprehension of Students Who Are Dysfluent Readers. Journal of Learning Disabilities, 42, 548-575. http://dx.doi.org/10.1177/0022219409338742

[23] Girbau, D. and Schwartz, R.G. (2008) Phonological Working Memory in Spanish-English Bilingual Children with and without Specific Language Impairment. Journal of Communication Disorders, 41, 124-145. http://dx.doi.org/10.1177/0022219409338742

[24] Siegel, L.S. (1988) Evidence That IQ Scores Are Irrelevant to the Definition and Analysis of Reading Disability. Canadian Journal of Psychology, 42, 201-215. http://dx.doi.org/10.1037/h0084184

[25] Ferrari, M. and Palladino, P. (2007) Foreign Language Learning Difficulties in Italian Children. Journal of Learning Disabilities, 40, 256-269. http://dx.doi.org/10.1177/00222194070400030601

[26] Carlson, S.M. and Meltzoff, A.N. (2008) Bilingual Experience and Executive Functioning in Young Children. Developmental Science, 11, 282-298. http://dx.doi.org/10.1111/j.1467-7687.2008.00675.x

[27] Bialystok, E. and Feng, X. (2008) Language Proficiency and Its Implications for Monolingual and Bilingual Children. Guildford Press, New York.

[28] Kohnert, K.J. and Bates, E. (2006) Balancing Bilinguals II: Lexical Comprehension and Cognitive Processing in Children Learning Spanish and English. Journal of Speech, Language, and Hearing Research, 45, 347-359. http://dx.doi.org/10.1044/1092-4388(2002/027)

[29] Perfetti, C.A. and Liu, Y. (2005) Orthography to Phonology and Meaning Comparisons across and within Writing Systems. Reading and Writing, 18, 193-210. http://dx.doi.org/10.1007/s11145-004-2344-y

[30] Kohnert, K., Yim, D., Nett, K., Kan, P.F. and Duran, L. (2005) Intervention with Linguistically Diverse Preschool Children: A Focus on Developing Home Language(s). Language, Speech, and Hearing Services in Schools, 36, $251-263$. http://dx.doi.org/10.1044/0161-1461(2005/025) 